\title{
Image compression using Super-Resolution Technique: A Review
}

\author{
Sudeepti Dayal \\ Dept. of ECE \\ T.I.E.I.T \\ Bhopal
}

\author{
Neelesh Gupta \\ Dept. of ECE \\ T.I.E.I.T \\ Bhopal
}

\author{
Neetu Sharma \\ Dept. of ECE \\ T.I.E.I.T \\ Bhopal
}

\begin{abstract}
With the rapid increasing demand of digital networks, people are likely to demand more and more accurate images which can be efficiently achieved using image compression. In image compression the irrelevant data is removed from the image keeping in mind that the data is stored in an adequate form. By doing this the data from the original image is sometimes is lost. Based on this, in the review paper the needs, its principle and various file formats of image compression are discussed. The paper also focuses on algorithms of image compression.
\end{abstract}

\section{Keywords}

Super resolution; TIFF; RAW; JPEG; DCT; Huffman; compression ratio.

\section{INTRODUCTION}

In digital imaging applications high resolution images or videos are used for image processing and analysis. Image resolution describes the detail an image has. Higher the resolution, higher will be the image details. The resolution may be classified as: spatial resolution, pixel resolution, spectral resolution, temporal resolution, and radiometric resolution. In this context, spatial resolution is used to differentiate between the low resolution and the high resolution images. Spatial resolution is the total number of independent pixel values to its unit length.

Another resolution technique which can be used to acquire the image details is the super resolution (SR) technique.

Super resolution is a technique that modifies the resolution of an image. The technique converts low resolution (LR) images to high resolution (HR) which in turn increases the high frequency components and removes the blurriness caused by the low resolution camera. Thus this technique is used to reconstruct the original image with the high resolution image given a set of observed images at lower resolution.

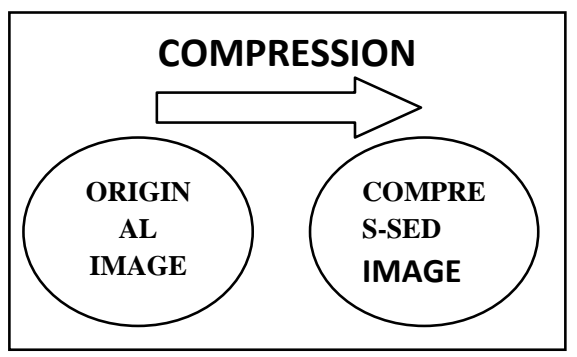

Fig 1 General Compression

\section{LITERATURE SURVEY}

In 2013 Gaurav Vijayvargiya, Dr. Sanjay Silakari, Dr. Rajeev Pandey represented various techniques of image compression [6]. In this they explained basic redundancies used to achieve compression. Along with that they focused on various lossless and lossy image compression techniques

B. C. Vemuri, S. Sahni, F. Chen, C. Leonard, J. Fitzsimmons presents lossless image compression [1]. They did an experimental evaluation using a number of image data sets.

S.Michael Vanitha, K.Kuppusamy in may 2013 surveyed on Fractal Image Compression [5]. The paper summarizes the method of one of the lossless image compression techniques, that is, fractal compression and hence concluded that on increasing the panel size, it will decrease the original file size and increases the image compression ratio. It will also decrease the processing time.

Ms. Pallavi M. Sune, Prof. Vijaya K. Shandilya presented image compression techniques based on wavelet and Huffman coding [7]. They discussed wavelet compression and image classification.

\section{NEED OF IMAGE COMPRESSION}

In general, data compression is the process of reducing the data needed to represent a given quantity of information. The goal of image compression is to diminish the data required to represent a digital image.

When a digital image is saved as a file on a web browser, it is basically saving it as a sequence of bits. Each pixel in the image consists of one byte and each byte is made from eight bits. If the image measures $\mathrm{M}^{*} \mathrm{~N}$ pixels, then $\mathrm{MN}$ bytes is needed to accumulate the image. There are 256 different bytes each of which has its own base 2 representation. So for a web browser to display a stored image, it must have the sequence of bits that describes the bit representation of each byte.

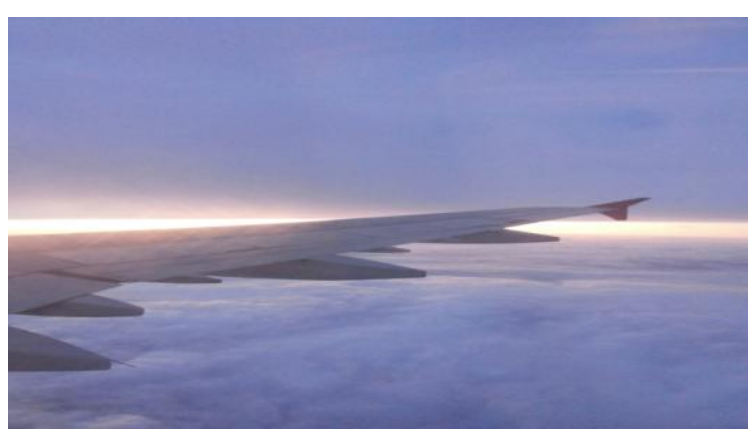

Original Image 


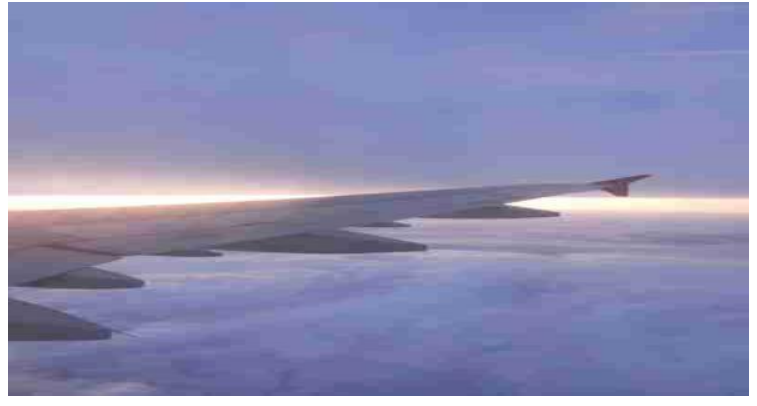

Compressed Image

Fig 2 Comparison of original image and compressed image

Table.1 Results showing variations in dimensions of original image to compressed image having $\mathbf{9 9 . 7 \%}$ savings in file size

\begin{tabular}{|c|c|c|}
\hline PARAMETERS & Original & Compressed \\
\hline Width & 2448 pixels & 400 pixels \\
\hline Height & 3264 pixels & 533 pixels \\
\hline File Size & $2,196.8 \mathrm{~kb}$ & $5.9 \mathrm{~kb}$ \\
\hline
\end{tabular}

\section{PRINCIPLE OF IMAGE OF COMPRESSION}

The purpose of image compression is to lessen the size of digital images to save storage space or transmission time. The image with dimensions $512 * 512 * 3=786,431$ pixels requires only 41,909 bytes when compressed in JPEG format. The question that arises is how the space can be saved while maintaining most of the content. The ratio of sizes tells us about the effectiveness the image. The term defining this is known as the compression ratio.

\section{Compression ratio:}

\section{Size of an original image}

\section{Size of the compressed image}

\subsection{Compression Algorithm}

\subsubsection{Lossless compression}

Lossless compression [1] is a compression algorithm in which the original image can be reconstructed from the compressed image i.e. after compression, reconstructed image is numerically identical to the original image. Some of the image files formats use lossless compressions are PNG or GIF.

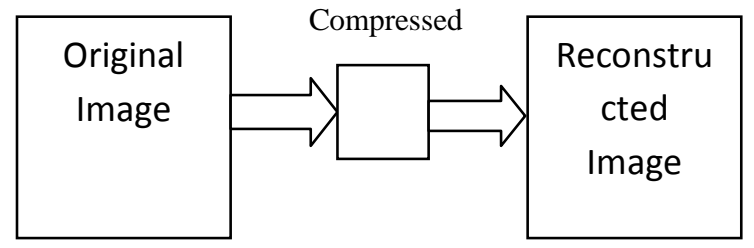

Fig 2 Lossless Compression

\subsubsection{Lossy Compression}

In lossy compression, redundant information is permanently removed. When an image is uncompressed, only a part of the original image exists and the rest is permanently removed which is unnoticeable to the user. Most commonly used image file formats include JPEG image file.

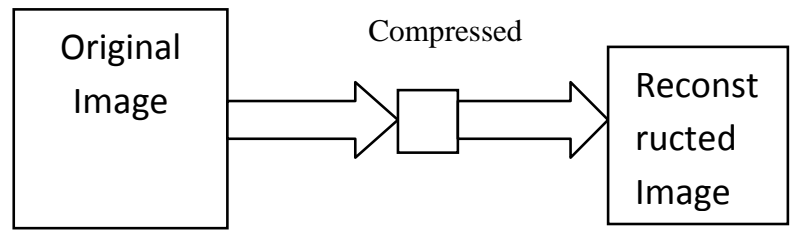

Fig 3 Lossy Compression

\subsection{Types of image file formats}

Images are classified as the following file formats:

\subsubsection{TIFF (Tagged Image File Format)}

The TIFF file format was created by Aldus (later on acquired by Adobe Systems). A file is described with file extension as ".tiff "or ".tif". The file format can be used as both lossless as well as lossy compression. HewlettPackard and Microsoft were the contributors to the format.

\subsubsection{PNG (Portable Network Graphics)}

Portable network graphics is raster graphics file format which supports lossless data compression. The format was retained by Unisys and is the widely used for transferring the images on the internet. PNG uses the file extension "PNG" or "png".

\subsubsection{JPEG (Joint Photographic Expert Group)}

This is the most commonly used format of lossy compression. JPEG uses the file extension as “jpg","jpeg","jpe". It has a maximum image size of $64 \mathrm{~K} * 64 \mathrm{~K}$ pixels and is used to store 24 bit photographic images.

\subsubsection{RAW}

RAW file format typically uses lossless as well as lossy compression technique. The format has the file extension ".raw". RAW file size is much larger than JPEG files but smaller than TIFF files. Advantage of RAW files is the color space can be set to any of the desired type. The RAW image files can be edited using Corel Paint shop Pro and Adobe Photoshop.

\subsubsection{GIF (Graphics Interchange Format)}

GIF is a bitmap image file format introduced by CompuServe. It is the second most widely used format in internet after JPEG. The format uses lossless compression technique and supports 8 bits each pixel and can use 256 maximum numbers of frames. GIF has a file extension as “gif”. GIF can be used for low resolution clips and short animations.

\subsubsection{BMP (Bitmap)}

Bitmap image file is a dot matrix data framework (raster graphics) image file format used to store bitmap images especially on operating systems and has a filename extension ".bmp". The bits per pixel of the bitmap image file determine the number of bits that each pixel has. When the field is equal to 1 that means the bitmap is a monochrome. When field is equal to 4 , the file has a maximum of 16 colors and when field is 8 it has a maximum of 256 colors. 


\section{COMPRESSION TECHNIQUES}

This section describes different techniques used in image compression [2] that are used for various applications in image processing.

\subsection{Lossless Image Compression}

The techniques used in lossless image compression are classified as:-

\section{Run length coding \\ 2. Huffman coding}

\subsubsection{Run length coding}

Run length coding is an algorithm which supports file formats such as BMP and TIFF. This coding is based on replacing a sequence of long string (pixel) to a sequence of shorter string (pixel). The run length code can be represented as a sequence $\{\mathrm{Ai}, \mathrm{Bi}\}$ where $\mathrm{Ai}$ is the intensity of pixel and $\mathrm{Bi}$ is the number of times it has occurred.

\begin{tabular}{|l|l|l|l|l|l|l|l|l|l|}
\hline 2 & 2 & 6 & 6 & 6 & 3 & 7 & 7 & 7 & 7 \\
\hline
\end{tabular}

Using run length coding: - $\{2,2\},\{6,3\},\{3,1\},\{7,4\}$

\section{Fig 4 Run length coding}

\subsubsection{Huffman coding}

David A. Huffman in 1951 created a compression algorithm completely lossless in nature. He was given the alternative of a team paper and then came up with the most efficient method of using a frequency sorted binary tree. He uses bottom up approach rather than using top down approach [7].

Consider a message consisting of 5 symbols:-

$\{$ ! @ @ \$ ! @ \% ! \$

This message is to be coded in such a way that it has minimum length.

Table.2 Huffman coding

\begin{tabular}{|c|c|c|}
\hline Symbol & Frequency & Code \\
\hline$!$ & 3 & 00 \\
\hline$@$ & 3 & 01 \\
\hline$\$$ & 2 & 10 \\
\hline$\%$ & 1 & 110 \\
\hline$\%$ & 111 \\
\hline
\end{tabular}

Length of the encoded message $=$

$3 * 2+3 * 2+2 * 2+\#+3=24$ bits

\subsection{Lossy image compression}

The techniques used in lossy image compression are classified as:-

1. Discrete cosine transform(DCT)

2. Fractal compression

\section{Fractional Talbot effect}

\subsubsection{Discrete cosine transform}

Discrete cosine transform (DCT) separates an image bands into sub-bands and is widely used image compression technique. It depicts an image as a sum of sinusoidal waveforms of changing magnitudes as well as frequencies. In DCT algorithm, the image is divided in to $8 * 8$ or $16 * 16$ blocks then discrete cosine transform is calculated for each of the block.

DCT Transmitter:-

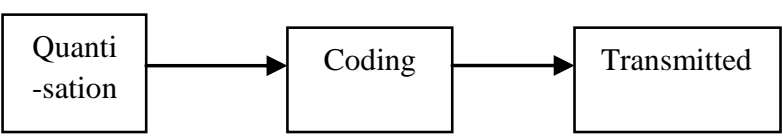

DCT Receiver:-

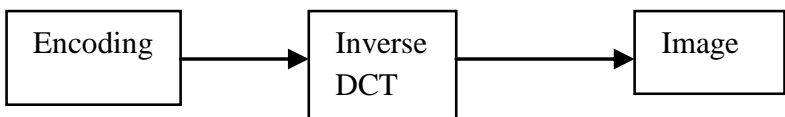

Fig 5 Transmitter and Receiver of DCT

\subsubsection{Fractal compression}

This technique runs in a loop mode that an initial image is formed from reduced copies of that image. The reduced image is the true replica of the original image. The figure describes the process of fractal compression [5].

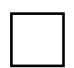

Original image

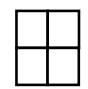

$1^{\text {st }}$ copy

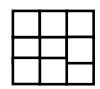

$2^{\text {nd }}$ copy
Fig 6 Fractal compression

\subsubsection{Fractional Talbot effect}

Fractional Talbot effect is a lossy compression technique which is widely applied in image processing and synthesis. The effect reproduces the periodic waves at periodic interval of time along the axis of propagation.

\section{CONCLUSION}

In this paper we reviewed image compression based on super resolution technique, need and principle. We also focused on various imaging file formats and compression techniques of lossy and lossless image compression. We thereby concluded that super resolution technique is the most efficient technique of image compression as it combines multiple low resolution images to form a high resolution image. The prime advantage of using super resolution technique is that it may costs less and the existing low resolution image can be still be utilized. The technique can be applied in the areas of satellite imaging, video imaging and medical imaging.

\section{REFERENCES}

[1] B. C. Vemuri, S. Sahni, F. Chen, C. Leonard, J. Fitzsimmons "lossless image compression".

[2] Sindhu M, Rajkamal R. "Image and its compression techniques". International Journal of Recent Trends in Engineering, Vol 2, No. 4, November 2009.

[3] Afshan Mulla, Namrata Gunjikar, Radhika Naik. "Comparison of Different Image Compression Techniques". International Journal of Computer 
Applications (0975 - 8887) Volume 70- No.28, May 2013.

[4] John Kerekes, "image data compression”. Rochester Institute of Technology.

[5] S.Michael Vanitha, K.Kuppusamy. "Survey on Fractal Image Compression". International Journal of Computer Trends and Technology (IJCTT) - volume4 Issue5-May 2013

[6] Gaurav Vijayvargiya, Dr. Sanjay Silakari, Dr.Rajeev Pandey. "A Survey: Various Techniques of Image Compression". International Journal of Computer Science and Information Security, Vol. 11, No. 10, October 2013

[7] Ms. Pallavi M. Sune, Prof. Vijaya K. Shandilya. "Image Compression Techniques based On Wavelet and Huffman Coding". International Journal of Advanced Research in Computer Science and Software Engineering, Volume 3, Issue 4, April 2013 ISSN: 2277 128X.

\section{AUTHOR'S PROFILE}

Sudeepti Dayal received her Bachelor's degree in Electronics and Communication Engineering from Sagar Institute of Research and Technology Bhopal, India in 2013.

Currently she is pursuing her master of technology in digital communication from Truba Institute of Engineering and Information Technology Bhopal, India. Her research interest is image compression. 